NISTIR 7662

\title{
QOD Standalone Users Guide
}

Julien Cuvillier

KC Morris

\section{NIT \\ National Institute of U.S. Department of Commerce} Standards and Technology 
NISTIR 7662

\title{
QOD Standalone Users Guide
}

\author{
Julien Cuvillier \\ KC Morris \\ Manufacturing Systems Integration Division \\ Manufacturing Engineering Laboratory
}

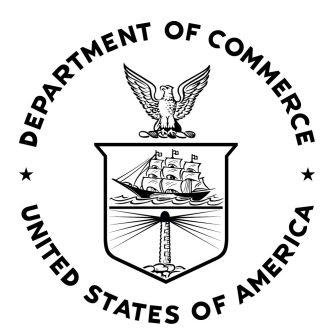

U.S. Department of Commerce

Carlos M. Gutierrez, Secretary

National Institute of Standards and Technology

Patrick D. Gallagher, Deputy Director 


\section{Abstract}

This report describes installation and use of the XML Schema [1] Quality of Design Standalone testing system, or QOD Standalone. QOD Standalone is one of several tools produced by NIST's XML Testbed project within the Manufacturing Systems Integration Division at NIST. These tools aid in producing high quality XML schemas using a standards-based approach to manufacturing systems integration.

QOD may be used by an XML schema developer to ensure compliance with a set of guidelines for XML schema development, a.k.a. Naming and Design Rules (NDR). QOD Standalone, derived from the original web-based system, may be used for off-line testing, hence the name "Standalone."

While the tool is configurable to use NDR from any number of original sources, it is distributed with tests of NDR from the Open Applications Group (OAG) [2] and Department of the Navy (DON) [3]. Tests for other NDR may be loaded into the system as they are available.

\section{Contents}

1 Introduction 4

1.1 About QOD Standalone . . . . . . . . . . . . . . 4

1.2 Background ........................ 4

1.3 Requirements ..................... 5

2 Getting started $\quad 5$

2.1 Starting QOD Standalone . . . . . . . . . . . 5

2.2 GUI Overview . . . . . . . . . . . . . . . . 6

3 Using QOD Standalone $\quad 7$

3.1 Test Cases selection . . . . . . . . . . . . . . . . 7

3.2 Schema selection and execution . . . . . . . . . . 7

3.3 Display Results . . . . . . . . . . . . . . . . . . . . . . 8

3.4 Export Results . . . . . . . . . . . . . . . . . . . . . . 8

3.5 Load and Save Results . . . . . . . . . . . . . . . . . . . 8

4 Example $\quad 8$ 


\section{Introduction}

\subsection{About QOD Standalone}

QOD Standalone is based on the Quality of Design tool for testing XML schemas and described in the Background section below. QOD Standalone is suitable for offline use and for users not wanting to share their tests or test results with others. The software is distributed with a set of tests based on rules from the Open Applications Group [2] and the Department of the Navy [3].

The web based version of QOD, available at http://www.nist.gov/qod, provides more functionality in terms of collaboration during use and development of rules. For further information about QOD or NIST see:

- The QOD web site: http://www.nist.gov/qod

Contact:xmlTestbed@cme.nist.gov

- The NIST web site: http://www.nist.gov

To learn more about the test materials used in this tool, see [4].

\subsection{Background}

A common problem in manufacturing systems integration is the need to exchange data between software applications. One technical approach to solving this problem is to use the W3C XML Schema Definition Language to define the data structure. This approach is being widely used in projects ranging from small activities involving only a single application to industrial and international standardization activities.

The XML Schema language is designed for describing and constraining the content of XML documents. It is well suited to the task of defining structured file formats for data definition, but as with any general purpose language a disciplined use of the language is needed to create a structure that is generally acceptable and reusable. Moreover, for consensus standardization activities, consistent use of the language is indispensible to achieving common understanding and agreement. To this end many organizations have taken the approach of defining how the language will be used for their community. The rules are written down in what is commonly called a "Naming and Design Rules (NDR)" document. 
Unfortunately, the proliferation of NDR documents can become a problem in itself for an XML user. The question becomes what NDR to use. The reason that there are so many different practices for how to use XML Schema is that there are different needs for different types of integration problems. What is perfectly reasonable and useful in one context may be problematic in another context. Reaching agreement on what is the right set of rules to use may be an endless task.

NIST is addressing this problem by providing tools that support the development and use of XML Schema including naming and design rules. There are several tools available. The XML Schema Qualify of Design (QOD) tool supports users in both specifying rules, enforcing those rules, and sharing those rules. QOD was originally developed as a web-based system. This is a stand-alone version of that system. The stand-alone system allows people to enforce selected rules on their own machines as they are developing the schemas.

\subsection{Requirements}

QoD standalone is fully cross-platform and requires Java 5.0 or higher (Available at http://java.com/en). It has not been tested with earlier Java versions. It also needs the following libraries:

- Saxon [5]: This library is provided with this software in the directory resources/lib/. The version used is 8 .

- JESS [6]: This library is not provided with this software. Download it and put the .jar files in the directory resources/lib or modify your classpath.

\section{Getting started}

\subsection{Starting QOD Standalone}

You can start QOD Standalone in the following ways:

- By double-clicking on QODStandalone.jar (Your system can be configured to open the jar as an archive, to launch QOD Standalone, right click $\rightarrow$ Open with $\rightarrow$ Java, or use the command line)

- The command line: java -jar QODStandalone.jar [myNDR.xml] 


\subsection{GUI Overview}

The GUI consists of one main window divided into 4 panes as illustrated in figure 1. The large pane on the right hand side of the screen contains a tree view of the rules and associated test cases from the NDR you have selected. The three smaller panes on the right hand side of the screen contain test results in various forms.

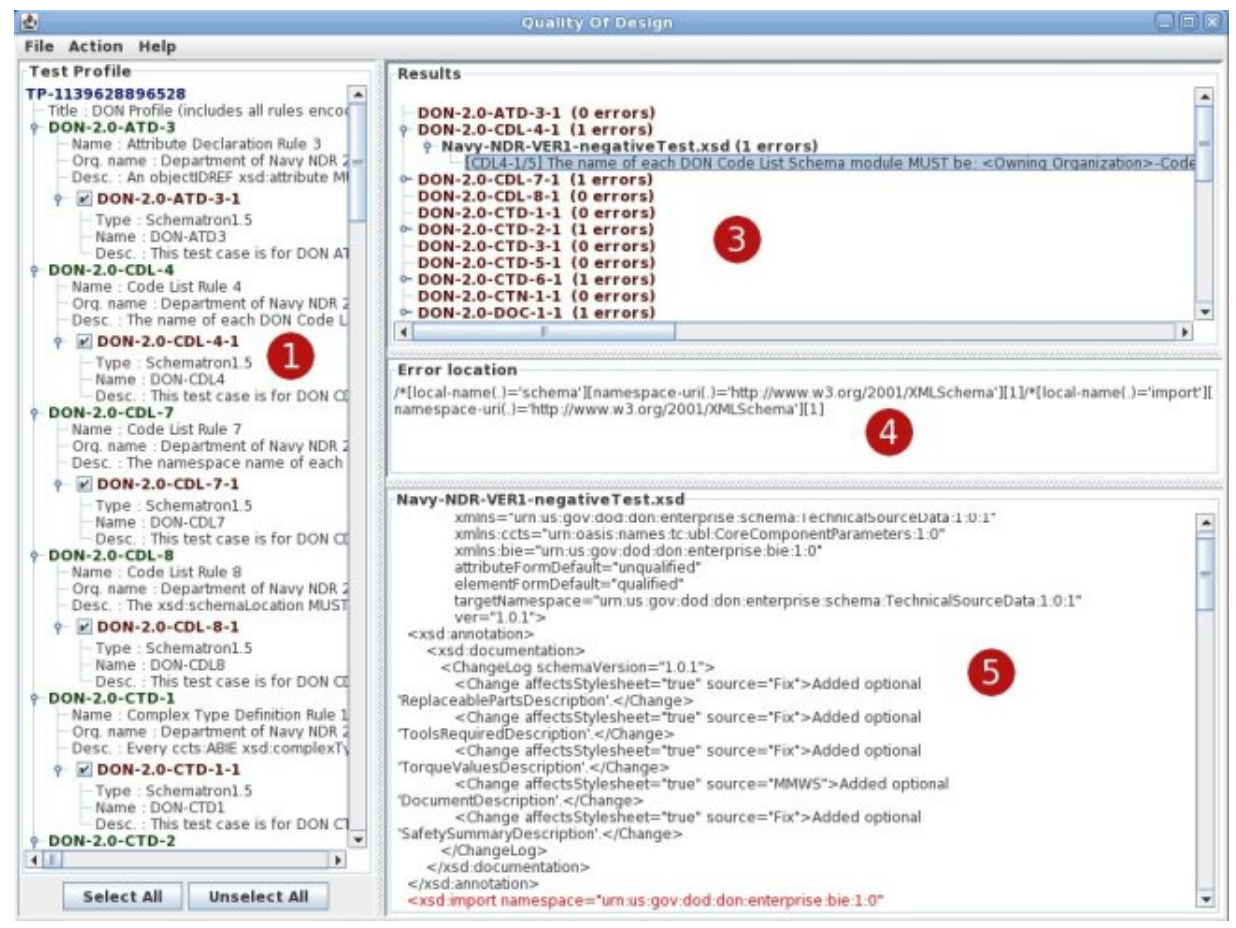

Figure 1: QOD Standalone User Interface

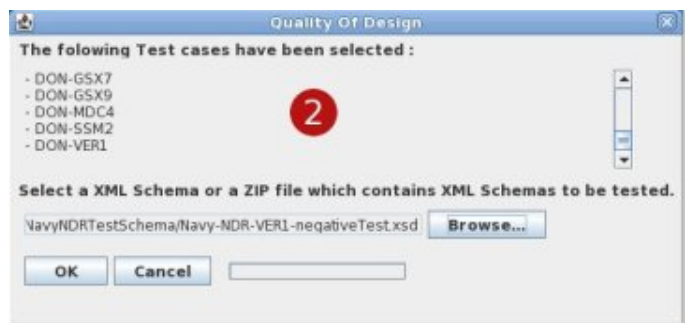

Figure 2: QOD Standalone Execution panel 
Panel 1. This is the Test Case selection area. Here you select the Test Cases you want to execute. By default, all the Test Cases are selected.

Panel 2. This window is the execution panel. You select the schema or the zip file containing the schemas you want to execute and launch the execution.

Panel 3. This area provides the results. The results are sorted by Test Case and by schema.

Panel 4. This area is used to provide the error's location as an XPath query [7].

Panel 5. This is the schema area. When an error is selected in the results tree, the error is highlighted here.

\section{Using QOD Standalone}

\subsection{Test Cases selection}

To select the Test Cases you want to use, first load an NDR or Test Profile (File $\rightarrow$ Open Test Profile). You can open the application with an NDRProfile already loaded by using the command line:

java - jar QODStandalone.jar myNDR.xml

A few profiles are provided with this software, others are available on the webbased QOD. An account is needed in order to export existing Test Profiles or to create a new one (For further information about NDRProfile see [8]). Then in the tree view select the Test Cases you want to execute. By default, all the Test Cases are selected.

- The symbol $\nabla$ indicates that a test case is selected.

- The symbol $\square$ is next to a test case that is not selected.

\subsection{Schema selection and execution}

To select the schemas to be tested, open the Execution Panel (Action $\rightarrow$ Test Schema). (You must have at least one Test Case selected.) Using the browse button, choose the schema file or the zip file containing the schemas to be tested. 
Click on the OK button of the Execution Panel to launch the execution. Click on the Cancel button to cancel it or to close the Execution Panel if no execution is running.

\subsection{Display Results}

The results are displayed in a tree view in the upper right hand panel. By clicking on a node in the tree, the error will be highlighted in the corresponding schema and displayed on the lower right hand side of the screen. The path will be provided in the middle section titled "Error location." The xpath can be copied and pasted from the screen into your own XML editor.

\subsection{Export Results}

The results can be exported in a file in a human readable way (Action $\rightarrow$ Export result). It contains the following information:

- Test Case ID and number of errors

- Error message

- For each Test Case, Schema name and number of errors

- Element name

\subsection{Load and Save Results}

All the files generated are deleted when the software is closed. So the results can be saved in order to be loaded in a new session (File $\rightarrow$ Save/Load results).

\section{Example}

This is an example of how QOD Standalone works. We will use one of the example rules provided.

The Test Requirement is DON-CDL7 from the Department of Navy the NDR [3] and the schema tested is a fabricated example schema. 


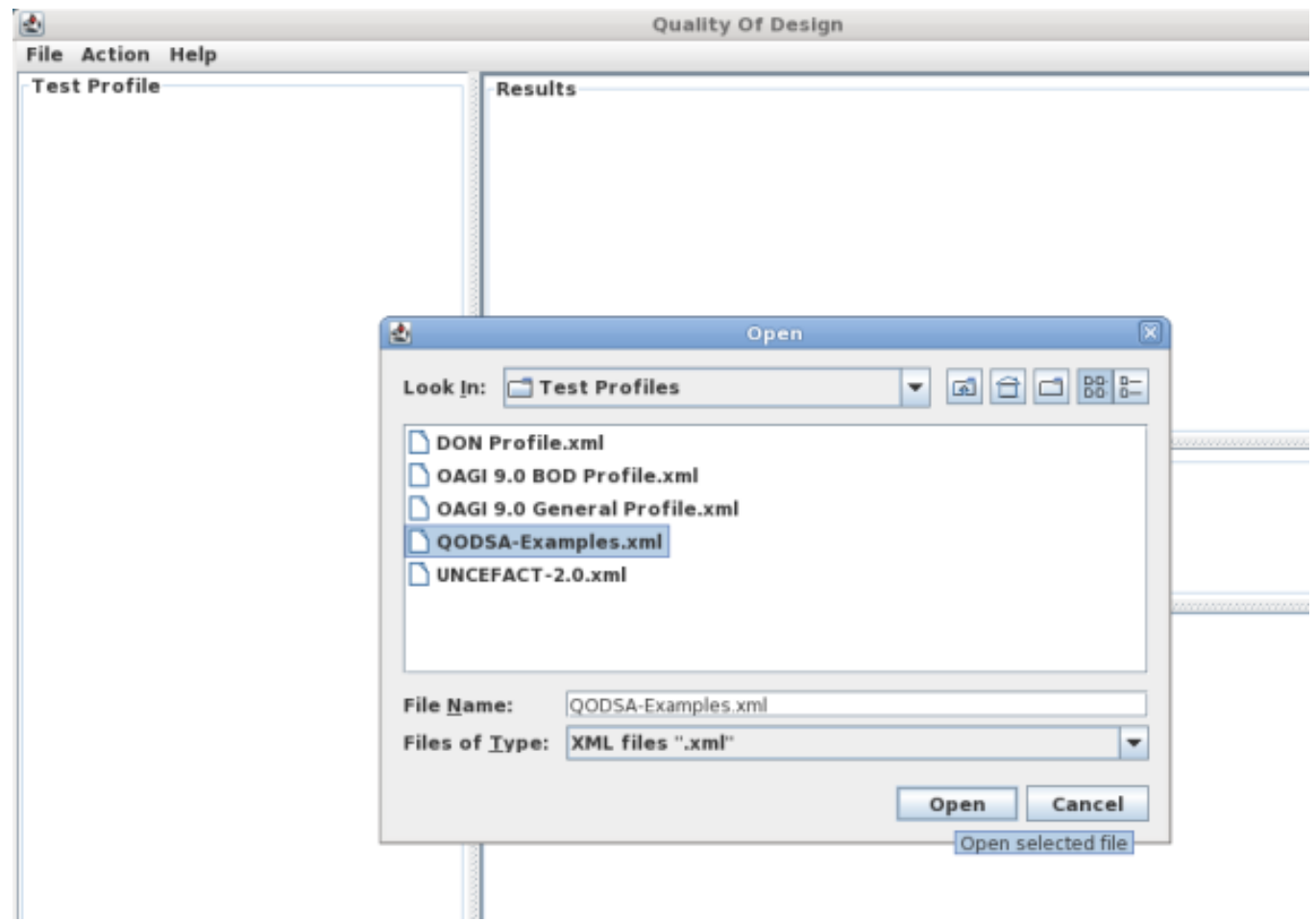

First we select the example Test Profile : QODSA-Examples.xml.

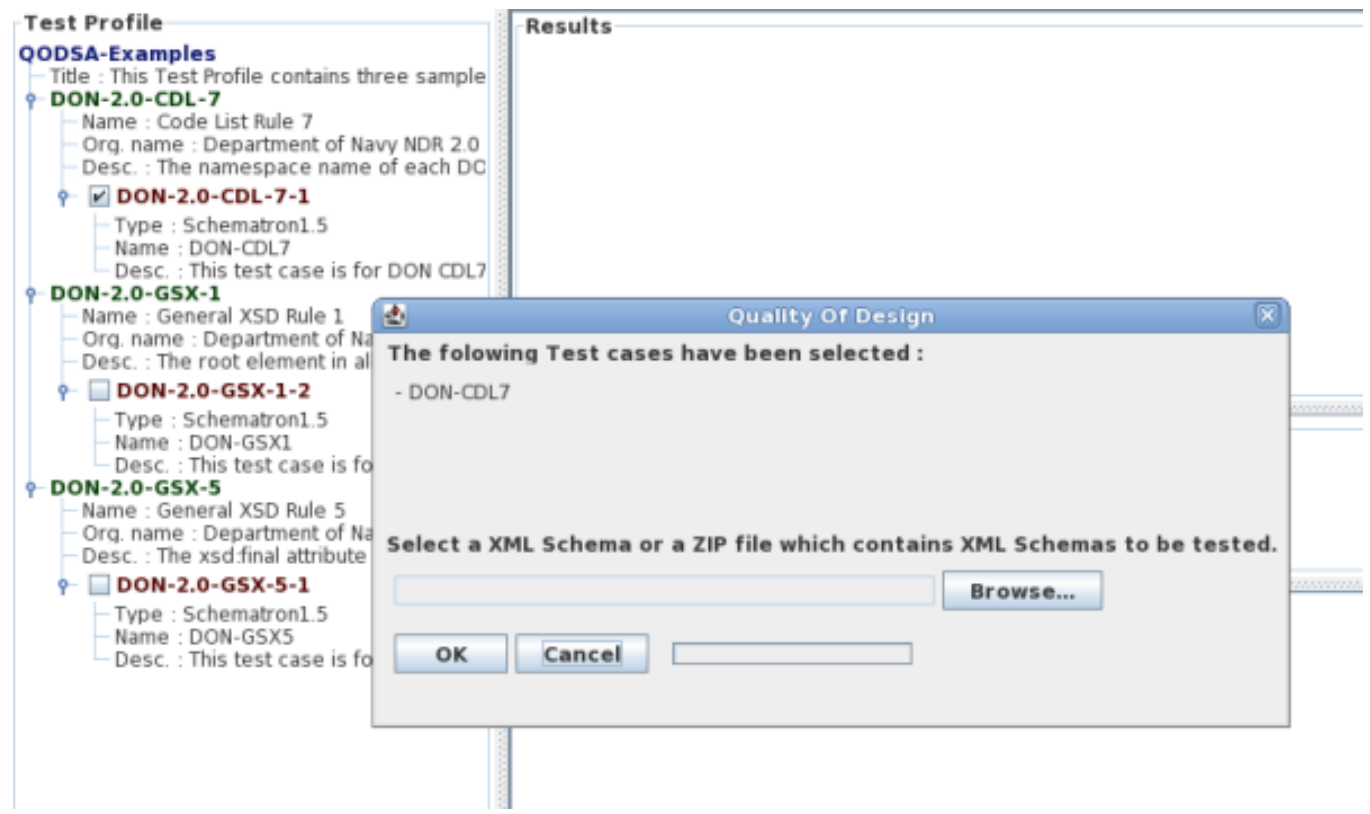

We select the single Test Case : DON-2.0-CDL-7-1. 


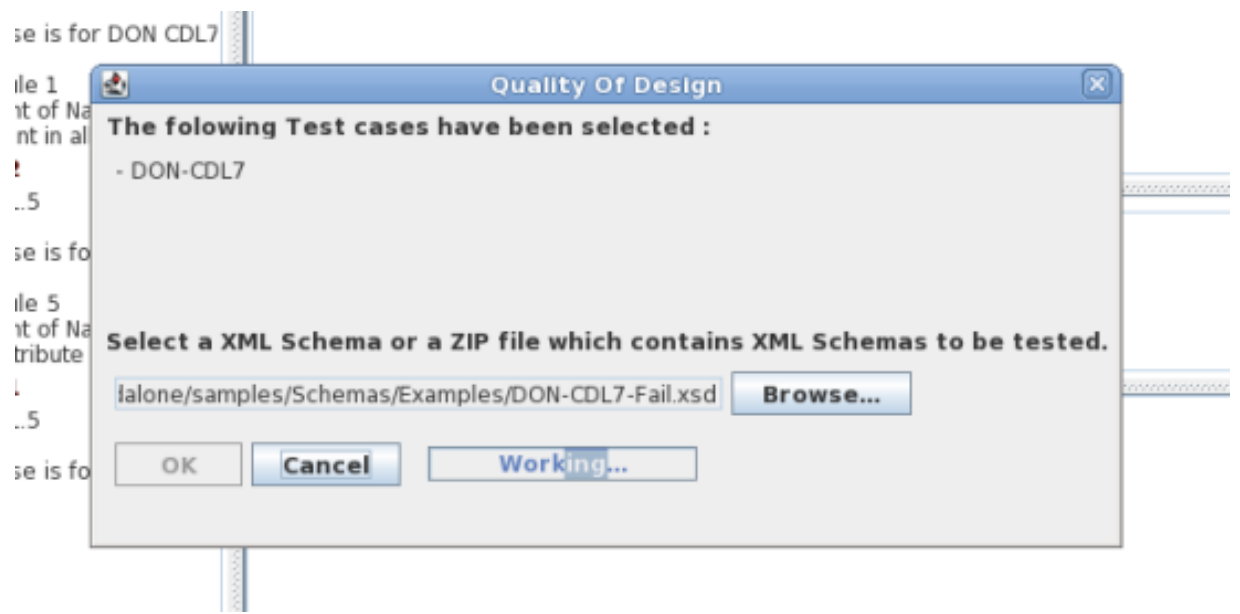

Then we select the corresponding schema in the samples directory and launch the execution.

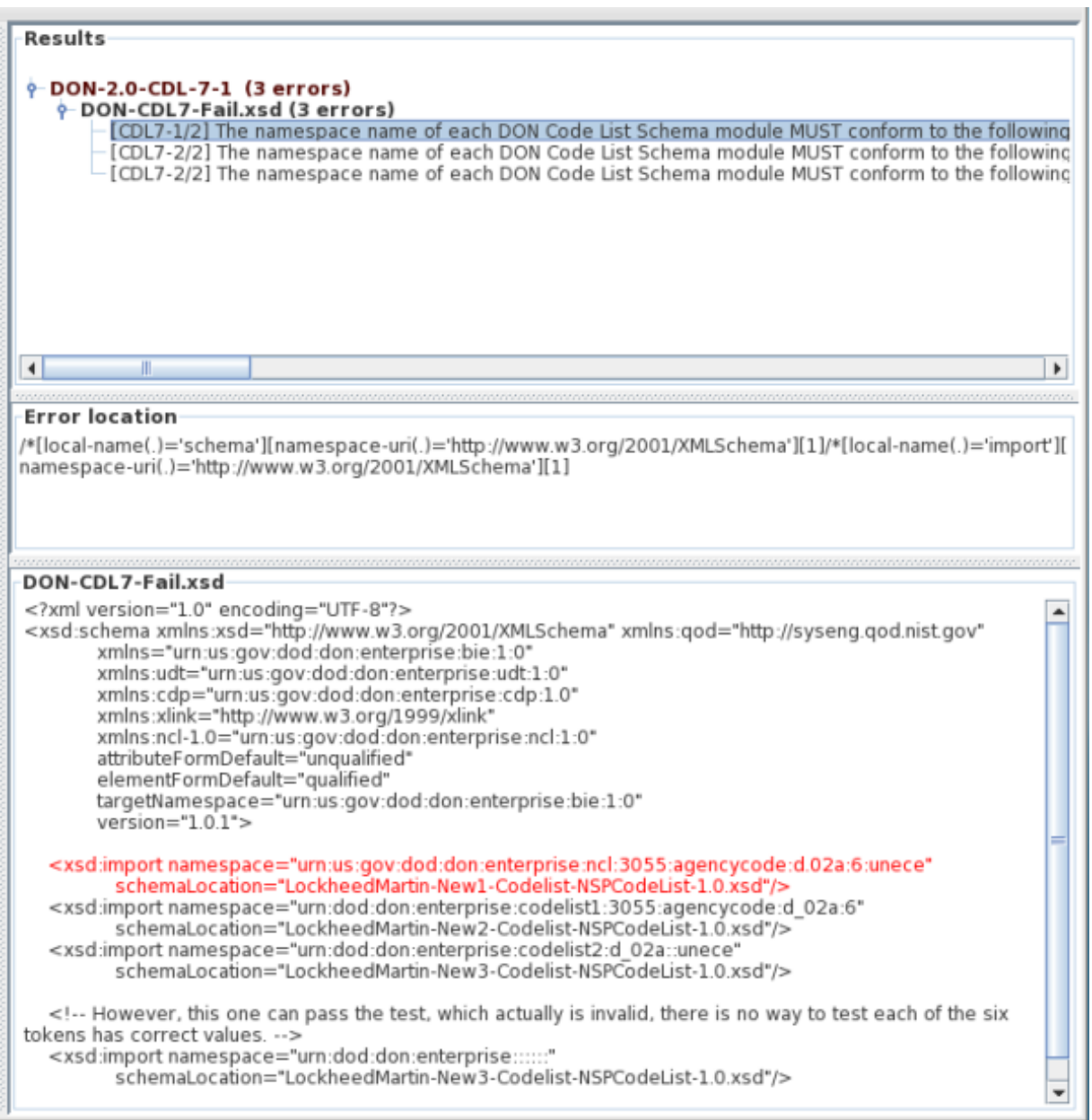


The errors appear in the tree view at the top of the screen. When we highlight the error by clicking on the line, the schema segment containing the error appears on the bottom of the screen with the error highlighted. Also the xpath expression to locate the error in the schema appears in the middle of the screen. This xpath can be cut and pasted into your favorite XML editor to locate the error in that editor. 


\section{Disclaimer}

Unless otherwise noted, this software was developed at the National Institute of Standards and Technology by employees of the Federal Government in the course of their official duties. Pursuant to title 17 Section 105 of the United States Code this software is not subject to copyright protection and is in the public domain. NIST's XML Testbed Tools are experimental systems. NIST assumes no responsibility whatsoever for its use by other parties, and makes no guarantees, expressed or implied, about its quality, reliability, or any other characteristic. We would appreciate acknowledgement if the software is used.

Links to non-Federal Government Web sites do not imply NIST endorsement of any particular product, service, organization, company, information provider, or content.

\section{References}

[1] World Wide Web Consortium. XML Schema. http://www.w3.org/XML/Schema.

[2] Open Application Group. OAGIS $\mathbf{9 . 0}$ Documentation. http://www .oagi.org/oagi/downloads/oagis/oagis9_doc.htm.

[3] Department of the Navy. Department of the Navy XML Naming and Design Rules Version 2.0, January 2005.

[4] Morris K. C., Frechette S. P., Goyal P., Lubell J., Kulvatunyou B., Sidi Malick S., Brayard N., and Tixier S. User's Guide for the Quality of Design Testing Tool and the Content Checker. NISTIR 7538, November 2008.

[5] Michael H. Kay. SAXON The XSLT and XQuery Processor. http://saxon. sourceforge.net/.

[6] Sandia National Laboratories. JESS, the Rule Engine for the Java ${ }^{\text {TM}}$ Platform. http://herzberg.ca.sandia.gov/.

[7] World Wide Web Consortium. XML Path Language Version 1.0. http://www.w3.org/TR/xpath.

[8] J. Lubell, B. Harvey, P. Goyal, and Morris K. C. NDRProfile Schema Version 1.0 User Guide. NISTIR 7547, December 2008. 